\title{
Detection of DNA Bases via Field Effect Transistor of Graphene Nanoribbon with a Nanopore: Semi-empirical Modeling
}

\author{
Asma Wasfi, Falah Awwad*, and Ahmad I. Ayesh
}

\begin{abstract}
DNA sequencing techniques are critical in order to investigate genes' functions. Obtaining fast, accurate, and affordable DNA bases detection makes it possible to acquire personalized medicine. In this article, a semi-empirical technique is used to calculate the electron transport characteristics of the developed z-shaped graphene device to detect the DNA bases. The z-shaped transistor consists of a pair of zigzag graphene nanoribbon (ZGNR) connected through an armchair graphene nanoribbon (AGNR) channel with a nanopore where the DNA nucleobases are positioned. Nonequilibrium Green's function (NEGF) integrated with semiempirical methodologies are employed to analyze the different electronic transport characteristics. The semiempirical approach applied is an extension of the extended Hückel (EH) method integrated with selfconsistent (SC) Hartree potential. By employing the NEGF+SC-EH, it is proved that each one of the four DNA nucleobases positioned within the nanopore, with the hydrogen passivated edge carbon atoms, results in a unique electrical signature. Both electrical current signal and transmission spectrum measurements of DNA nucleobases inside the device's pore are studied for the different bases with modification of their orientation and lateral translation. Moreover, the electronic noise effect of various factors is studied. The sensor sensitivity is improved by using nitrogen instead of hydrogen to passivate the nanopore and by adding a dual gate to surround the central semiconducting channel of the $z$ shaped graphene nanoribbon.
\end{abstract}

Index Terms- DNA sequencing, electronic transports, graphene nanoribbon, nanopore, quantum transport.

The article is submitted in December 2020. This research is supported by United Arab Emirates University with Fund number 31R128. Falah Awwad (f_awwad@uaeu.ac.ae) is the corresponding author of this research.

Asma Wasfi is a PhD student at Electrical Engineering Department, United Arab Emirates University, P. O. Box 15551, Al Ain, United Arab Emirates (e-mail: 201180954@uaeu.ac.ae).

Falah Awwad* is a Professor with the Electrical Engineering Department ,United Arab Emirates University, P. O. Box 15551, Al Ain, United Arab Emirates (e-mail: $f$ awwad@uaeu.ac.ae).

Asma Wasfi and Falah Awwad are with Zayed Center for Health Sciences, United Arab Emirates University, United Arab Emirates.

Ahmad I. Ayesh is a Professor with the Department of Math., Stat. and Physics, Qatar University, Doha, Qatar (e-mail: ayesh@qu.edu.qa).

\section{INTRODUCTION}

$\mathrm{D}$ NA sequencing is an essential technology with the general goal to discover and cure diseases [1]. The DNA signature or sequence is unique for each individual. Detecting the DNA sequence makes it possible to find out the cause and cure the diseases people could have in their future. Sequencing the DNA helps translate genetic data into clear answers and thereby which enables people to make clear decisions built on their genetic prepositions and risks. Therefore, cheap, reliable, and fast DNA sequencing approaches leads to different applications in personalized medicine and genetics subfields. Various research works have been generated by researchers to develop sensors that can acquire DNA sequence accurately and cheaply. DNA sequence and structure design can be facilitated by software named DNA shop which provides a better idea about the designed DNA sequence before the time consuming laboratory experiments [2].

Several nanopore techniques have been initiated and studied to acquire reliable and successful DNA sequencing [3]. Nanopore-based sequencing applications were initiated in 1995 [4]. The nanopore techniques have the potential to achieve low cost and fast DNA sequencing by removing the necessity for enzyme dependent amplification and fluorescent labeling. Two essential categories of the pores are utilized for the third generation devices to detect the DNA sequence: (i) solid-state pores, and (ii) protein pores [5].

Previous theoretical works showed that transverse tunneling current passing through the electrodes on the nanopore helps to differentiate between the DNA bases due to the variation of the bases electronic structure [6-9]. Lindsay's experimental work resulted in an enhancement in DNA bases detection [10, 11]. One of the DNA detection techniques Lindsay used relies on using two gold electrodes with a DNA base placed between them where the current passing through the nucleotides is used to identify the sequence [12]. Lindsay's group recognition tunneling method depends on using a pair of electrodes where one of the electrodes has sensing chemicals and the other has the target nucleotide to be detected. In this method, each one of the DNA nucleobases generates unique tunneling current signature which makes it possible to identify the DNA sequence $[11,12]$. However, this technique might face the problem of interference of adjacent bases if they are in between the electrodes simultaneously where more than one 
type of bases contributes to the signal. It was noticed that functionalizing the pore-edge improves the sensitivity of graphene monolayer with a pore. Paulechka et al. found that graphene nanopore functionalized with cytosine enhances guanine identification accuracy by $90 \%$ [13].

Choosing the material for nanopore based sensor fabrication to detect DNA bases has critical considerations. Because of graphene's unique structure and characteristics [14], various graphene based sensors were developed and studied both experimentally and theoretically [15]. Graphene electron transport properties are attracting researchers' interest [16-18]. Graphene layer depth is similar to the dimensions of DNA nucleobases' [14].

Graphene nanoribbons (GNR) are categorized into two types based on their cut pattern such as AGNR and ZGNR. ZGNR is metallic whilst AGNR can be either metallic or semiconducting according to the number of carbon chains [19]. AGNR that has a number of atoms of $\mathrm{Na}=(3 n+2)$ width where $n$ is a positive integer is metallic [19]. The GNR is considered a better choice than graphene junctions for transport requirements due to its properties [19]. ZGNR is less sensitive to nanopore shape than AGNR.

Graphene is considered an ideal membrane for nanoporebased DNA detection due to its structure and thinness. Different approaches utilize graphene's special properties to find out the DNA sequence [15]. The main methods for DNA detection based on graphene are: (i) measuring the current alteration within a pore in a graphene layer, (ii) measuring the modulation in tunneling current through a nanogap between a pair of graphene electrodes, (iii) in-plane alteration in current for a graphene nanoribbon, and (iv) variation in graphene current due to DNA nucleobases physisorption onto graphene [15]. Graphene-based nanopore sensors are promising for DNA detection. Using a multilayer grapahene nanopore has some advantages. For example, bilayer graphene was utilized to find out the DNA bases which resulted in less fluctuation of the DNA bases within the nanopore [20]. Nanopore-based sensors were used by various groups of researchers to find out the DNA sequence experimentally where the pore supporting membrane was graphene [21-23]. Moreover, DNA current can be detected to find out the sequence by using transverse electrodes of graphene [24]. However, using a nanogap of graphene enables multiple bases of DNA to go through which will result in interference in the readings of ionic current of the bases. Thus, graphene nanopores are better than graphene nanogaps.

The electric field effect for graphene sheets can be enhanced by placing a gate potential which enhances the sheet mobility. Graphene nanoribbons are very promising and can be used in multi-terminal devices. Various graphene based devices are configured such as: patterned graphene devices and gating devices [25].The electron density of state is critical in measuring the optical and electrical characteristics of these devices [25]. Graphene has various unique mechanical, optoelectronic, electrical characteristics. The nanoribbon size, shape, and spectral broadening play an important role in these characteristics [26]. Graphene is being highly used for field effect transistor (FET) fabrication due to its great mobility of both holes and electrons as well as distinctive band structure [27] . Its electrical conductance provides electrical reaction signal to DNA nucleobase translocations [28], where theoretical investigations revealed that each nucleobase interaction with the material of graphene results in a sole electrostatic voltage signal [29]. Furthermore, the high conductance of graphene enables high current as compared with ionic current [28, 30, 31]. Therefore, graphene based FETs are anticipated to offer high signal to noise ratio and fast sequencing $[28,32]$.

Recently, it is becoming highly important to study the single atom effect of electronic devices by using simulation tools. Different electron transport simulators models which are based on NEGF are developed. These models are divided into two categories: ab-initio methodologies [33, 34], and semiempirical methodologies $[35,36]$. An essential feature of the semi-empirical methodologies are their lower computational expenses [37].

In this paper, the electron transport properties and the interactions among the Z-shaped device and the DNA bases are studied using NEGF integrated with SC-EH. Every individual DNA base is positioned within a pore with the passivation of its edge carbon atoms using hydrogen (H-pore) or nitrogen (N-pore) to generate the transport characteristics of the DNA nucleobases. Our work reveals that each one of the four bases leads to a specific current range which helps in differentiating among the different DNA bases. The transport characteristics of the DNA nucleobases inside the pore are studied after adding a dual gat to surround the center of the $\mathrm{z}$ shaped nanoribbon. The sensor sensitivity is improved by adding the dual gate. This study extends the work in [38] by adding a dual gate terminal to surround the semiconducting channel to improve the sensor signal. The sensor's current and transmission spectrum for each DNA base at room temperature are analyzed. The sensor sensitivity in this work is also improved by using nitrogen instead of hydrogen to passivate the nanopore edge carbon atoms in the z-shaped graphene nanoribbon in previous work [39]. Moreover, various noise factors such as DNA backbone, solution, nearest neighbor are studied.

\section{SENSOR CONFIGURATION}

This study investigates the z-shaped device behavior in detecting the DNA bases, by using semi-empirical simulations. Figure 1(a) displays the nanoscale sensor configuration (without a gate), while Figure 1(b) shows a graphene nanoribbon field effect transistor. The sensor is built of three terminals: drain, source, and dual gate. The gate potential makes it possible to measure the sensor current and transmission spectrum where the gate is expected to enhance the sensor sensitivity. A $10.1 \AA$ nanopore is initiated in the center of the sensor channel. The z-shaped transistor consists of a pair of electrodes made of metallic ZGNR connected with a semiconducting AGNR channel. Thirteen carbon chains are used to build the AGNR channel in width and sixteen carbon chains are used to build each of the ZGNR electrodes. (Width 
of the AGNR channel is $16.6 \AA$ while the ZGNR electrodes length is $15 \AA$ ). Two gates are added where each gate consists of dual layers: metallic and dielectric where the constant of the dielectric layer is 4 . One gate is placed under the central AGNR and the other gate is added above the channel. The carbon atoms edges are saturated by bonding along neighbor atoms. The nanoribbon carbon atoms edges are saturated by hydrogen or nitrogen. The cross section of the dual gate zshaped graphene nanoribbon FET (DG-ZGNR-FET) sensor is shown in Figure 2.

(a)

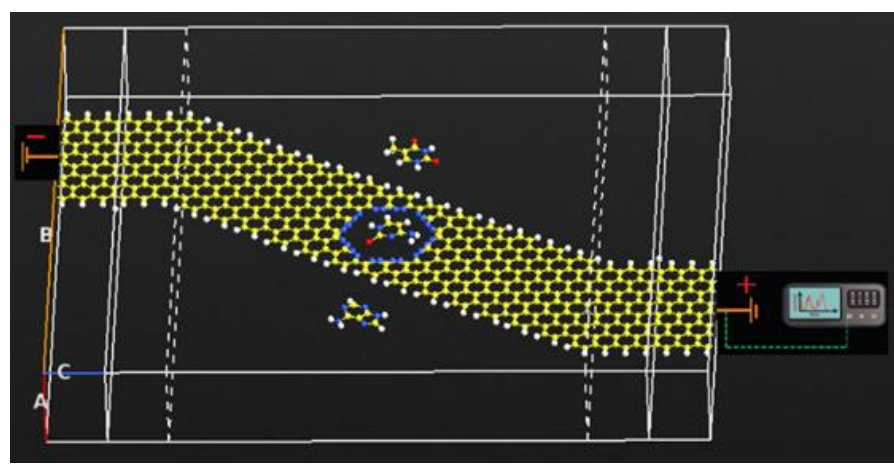

(b)

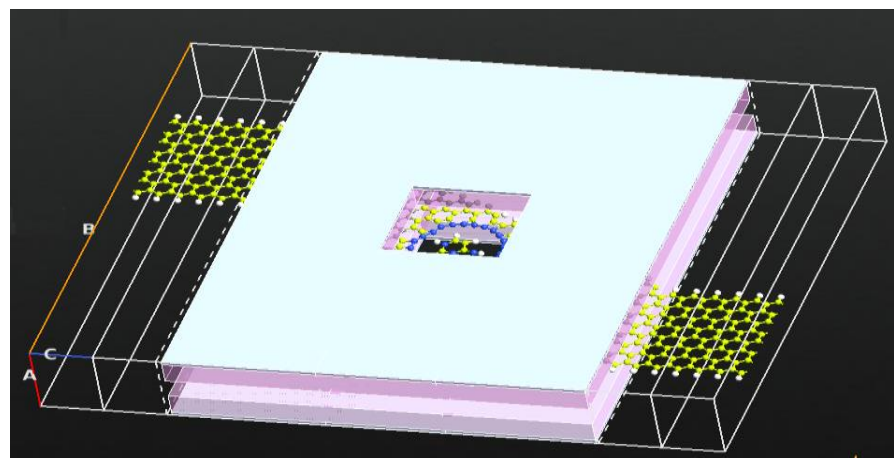

Figure 1. (a) Schematic diagram of $\mathrm{z}$-shaped GNR with a single DNA base passing within the pore. The edge carbon atoms of graphene nanopore are passivated with nitrogen while the edge carbon atoms of the GNR are passivated with hydrogen. (b) Schematic figure of the z-shaped FET with a pore. DNA bases go across the pore while the transverse electronic current is passing across the graphene sheet. The dual gate is biased at $1 \mathrm{~V}$ each side and the bias potential is fixed among the source and drain $(+1.4$ and $-1.4 \mathrm{eV})$. Color code: hydrogen-white, carbon-yellow, oxygen-red, and nitrogen-blue.

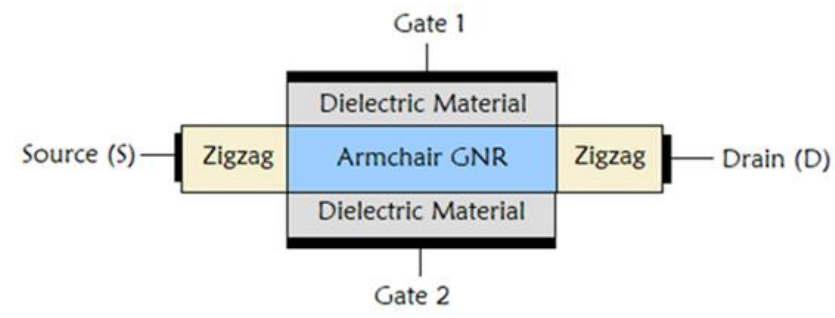

Figure 2. Cross-sectional view of the DG-ZGNR-FET sensor. The designed sensor is made of two electrodes and a semiconducting channel surrounded by two gates.

This study is a proof of concept that the designed z-shaped
GNR transistor can be used for DNA bases detection. This work includes four major enhancements over the previous work [39]. The first improvement is utilizing semi-empirical model as an alternative of first principles model. The semiempirical approach is presented to calculate the atomic scale electron transport properties. It was demonstrated by Stokbro et al. [37] that semi-empirical model measurements are in closer consent with experiments than first principles approach. Moreover, semi-empirical model can be used in parallel with experiment where the model can be fitted to get accurate results. The primary advantage of using semi-empirical model is the less computational expenses. Moreover, for many systems the unoccupied levels' energies are poorly modeled within DFT and require large number of parallel computers to perform the computation which is unfeasible. Stokbro et al. illustrates that semi-empirical model can give accurate results for a wide range of nanoscale sensors including $\mathrm{z}$-shaped graphene transistor with the advantage of the high computational speed and the less cost $[37,40]$. Also, semiempirical models are more flexible and generate good description of molecular conduction while first principles are not flexible to handle the uncertainties of electronic structure or the complicated three terminal devices [41, 42]. The second enhancement is the addition of dual gate which enhances the graphene nanoribbon sensor mobility [40]. The gate controls the current flow across the transistor based sensor where a small gate potential amplifies and controls the device current. The gate potential modulates the semiconducting channel charge density and enhances the flowing current through the transistor. The higher sensor current is expected to enhance the sensor sensitivity for DNA detection [28]. The third improvement is using nitrogen to saturate the nanopore instead of hydrogen. The nitrogen is considered as n-type donor. The nitrogen passivation was proofed to be electron rich resulting in n-type transistor behavior [43, 44]. The nitrogen passivation results in excess free electrons which results in higher sensor current and better sensor performance. Nitrogen passivation makes the sensor highly sensitive to the single molecule and to the intramolecular electrostatics within the molecule [43-45]. The fourth enhancement is studying the effect of noise factors such as sugar-phosphate backbone, nucleobases' translation, solution, and the neighboring nucleotides.

ATK-VNL simulator provides ATK-semi-empirical which is used to analyze the transmission spectrum and electrical current of the different DNA bases within the graphene nanopore. Transport characteristics for the various bases are investigated with variation in their orientation and lateral translation. The main interest in this study is to find out the relevant current for each DNA nucleobase to get a specific signature to identify the DNA bases.

\section{Computational Methodology}

Before generating the electronic transport measurements, the $\mathrm{z}$-shaped device and nucleobases were optimized and relaxed using the density functional theory until the separate atomic force was lower than $0.05 \mathrm{eV} / \AA$. The cut off energy is fixed at $400 \mathrm{eV}$ to expand the orbitals of Kohn-Sham (KS). Perdew-Burke-Ernzerhof parametrization was utilized for the exchange correlation function [46] . The different electronic 
characteristics including transmission spectrum and current were investigated using NEGF combined with semi-empirical modeling.

In order to generate the charge density, a 10 Hartree mesh cut-off was fixed. The sampling k-points for the Brillouin Zone integration are $2 \times 2 \times 100$ k-points. Poisson equation with marginal conditions was used where the electrostatic potential boundary condition of electrodes in the $\mathrm{C}$ direction was selected as a Dirichlet condition and the other two directions (A, B) were selected as a Neumann boundary condition. The Neumann boundary condition was employed to make sure that the electrostatic voltage was fixed and results in a zero derivative. This was a proper boundary condition whenever the transistor had a gate made of metal [47].

The sensor transverse current was generated from the transmission spectrum by NEGF technique [48]. During the single stranded DNA translocation across the sensor's pore, different DNA bases' orientations were employed. Therefore, it was highly important to take into account the bases' orientation influence on the current and transmission spectrum.

Calculation of the zero bias transmission spectrum between the electrodes (source and drain) was carried by the following equation $[30,49]$ :

$T(E)=\operatorname{Tr}\left\{\Gamma_{D}(E) G(E) \Gamma_{S}(E) G^{\dagger}(E)\right\}$

where, $E$ represents the energy, $\operatorname{Tr}$ refers to the trace, $\Gamma_{D_{s} S}(E)=i\left[\sum_{L_{s} R}(E)-\sum_{S_{s} D}^{\dagger}(E)\right]$ represents the broadening level resulting from the source and drain coupling, and $\sum_{L_{s} R}(E), \Sigma_{S_{s} D}^{\dagger}(E)$ are the self-energies produced by the drain and source. The bias transmission spectra was measured by NEGF approach, as integrated in ATK-VNL, using [49]:

$T\left(E, V_{b}\right)=\operatorname{Tr}\left\{\Gamma_{D}\left(E, \mathrm{~V}_{D}\right) G(E) \Gamma_{S}\left(E, \mathrm{~V}_{S}\right) G^{\dagger}(E)\right\}$

where, $\mathrm{G}$ and $G^{\dagger}$ are correlated to the major scattering region

advanced Green's function, and $V_{b}=V_{s}-V_{d}$ where $V_{b}$

refers to the bias voltage among drain and source. D, S, R, L represents drain, source, right, and left respectively. The semiinfinite influence of the source and drain was calculated by establishing the self-energies $\sum_{L_{x} R}(E)$ and $\sum_{S_{x} D}^{\dagger}(E)$ in the

effective Hamiltonian.

The $\mathrm{T}(\mathrm{E}, \mathrm{V})$ integration through the energy window was measured by utilizing the variation of the Fermi functions $f_{S_{x} D}(E)=\left\{1+\exp \left[\left(E-E_{F}-e V_{S_{s} D}\right) / k_{B} T\right]\right\}^{-1}$

that gave the overall current [49]:

$$
I=\frac{2 e}{h} \int_{-\infty}^{\infty} d E T(E, V)\left[f_{S}(E)-f_{D}(E)\right]
$$

\section{Results ANd Discussion}

Each of the different types of DNA nucleobases inside the nanopore leads to a sole variation in the device current and transmission spectrum. The results of translocation of DNA bases are as below.

\section{A. Transmission Spectrum}

The z-shaped device transmission spectrum is calculated with 2, 2, 100 sampling point. The energy domain -2 to $2 \mathrm{eV}$ has 200 sampling points. Cerda. Carbon (graphite) basis set is chosen for carbon, while Hoffman is chosen for the rest of the atoms [47].

Figure 3 shows the zero bias transmission spectrum for the developed sensor of $1.01 \mathrm{~nm}$ pore with two types of pores based on the passivation of the edge carbon atoms: hydrogen (H-pore) or nitrogen (N-pore). The figure reveals low values of transmission spectrum within the energy range $[-0.7,1.1]$ $\mathrm{eV}$, which is due to the band gap energy window within the AGNR channel. The number of transmission peaks produced by $\mathrm{N}$-pore is more than those produced by $\mathrm{H}$-pore. The $\mathrm{N}$-pore transmission spectrum indicates higher sensor current than the H-pore sensor.

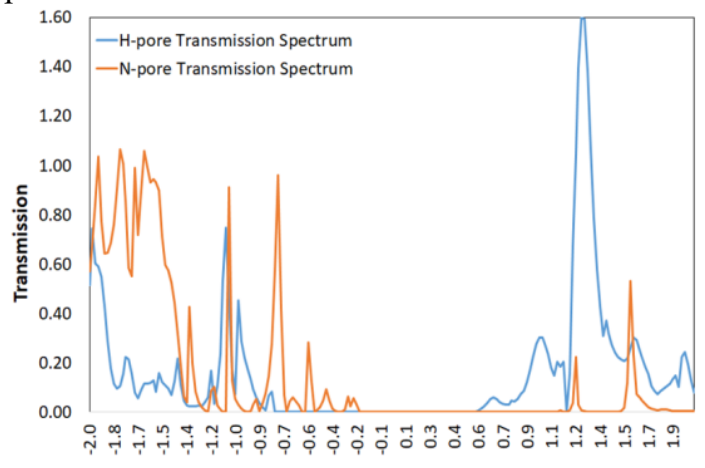

Energy (eV)

Figure 3. The bias transmission spectra at zero for z-shaped device with an empty (H-pore) or (N-pore).

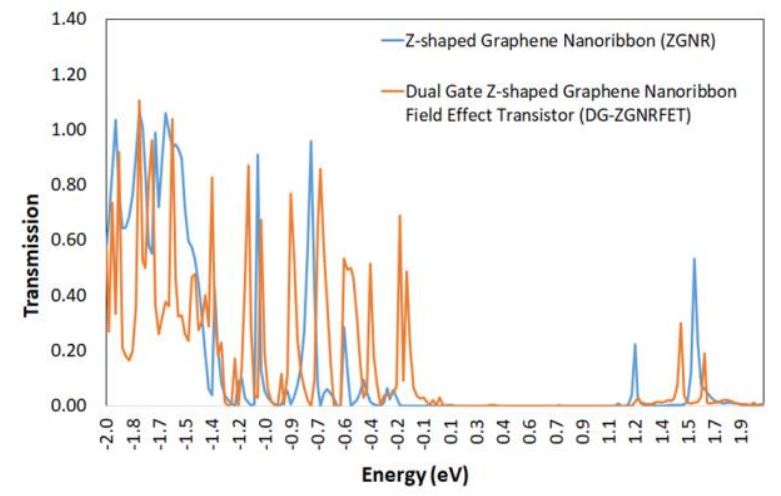

Figure 4. The bias transmission spectra at zero for both z-shaped graphene nanoribbon and dual gate $\mathrm{z}$-shaped transistor with $\mathrm{N}$-pore.

Figure 4 displays the zero bias transmission spectra for comparison between the $\mathrm{z}$-shaped graphene nanoribbon and the DG-ZGNR-FET. The DG-ZGNR-FET has more transmission peaks with higher intensity than the $\mathrm{Z}$-shaped graphene nanoribbon which indicates higher sensitivity and higher current readings as compared with the zero bias transmission.

Figures 5(a)-5(d) display the transmission spectra for T, C, $\mathrm{G}$ and $\mathrm{A}$ bases under a fixed bias voltage of $2.8 \mathrm{~V}( \pm 1.4 \mathrm{~V}$ on each source and drain). These figures reveal that each base has a unique transmission spectra leading to a unique current. Each panel reveals the transmission spectrum for four 
orientations for each base. This reveals that there is a slight difference in the transmission spectrum for every base due the various orientations which results in a slight difference in the current value. Figure 6 shows the structure of the four different types of DNA bases at $0^{\circ}$ rotation. The structure of the rotated Adenine base is shown in Figure 7. Each DNA base is rotated at an angle of $180^{\circ}$ with respect to the $\mathrm{x}$-axis, xz-plane, and xy-plane.

There are two categories of DNA bases: pyrimidine bases including cytosine and thymine and purine bases including guanine and adenine. The main distinction among pyrimidine and purine nucleobases is their size. One can understand from the transmission spectra of the various bases, that the differences in the chemical and physical structure of purine and pyrimidine bases influence the sensor transport characteristics differently, which make it possible to distinguish the two different DNA groups due to fixed bias voltage. Purine base has two carbon-nitrogen rings, while pyrimidine has one carbon-nitrogen ring which result in higher current for purines.

\section{B. Transverse Current}

Each DNA base local electronic densities of states is unique and can be used to differentiate between them when translocated across the pore. Figure 8 shows the $z$-shaped sensor transverse current for each base when placed inside the center of the H-pore or N-pore. Utilization of N-bond can improve the transverse current rate compared with H-bond. Therefore, N-pore can be utilized as an alternative of H-pore to improve the sensor sensitivity to identify the DNA bases. Herein, N-bonds improve DNA bases and the device coupling which increases the transverse current magnitude. As a result, the current measurability and detection are clearer and highly enhanced. Moreover, the read speed of DNA sequence is increased due to the high current, thus speed up the detection process.

Figure 9 displays the transverse current for the various types of DNA nucleobases in the nanopore of the two terminal and three terminal sensors. A $1 \mathrm{~V}$ gate potential is applied for each gate of the DG-ZGNR-FET sensor across the central region, and the voltage between the source and drain is fixed as +1.4 and $-1.4 \mathrm{~V}$, where the device functions as a field effect transistor. The sensor with a gate voltage exhibits higher transverse current rates and thus higher sensitivity as compared with the case without gate. Figure 9 shows the result for one orientation $\left(0^{\circ}\right.$ angle) for each base within $z$-shaped graphene nanoribbon pore and the DG-ZGNR-FET pore. The higher sensitivity is referred to the higher current reading which is expected to be higher than the noise and leads to faster sequencing speeds.

Figure 10 displays the possible current ranges when the nucleobases are rotated at $180^{\circ}$ angle with respect to the $\mathrm{x}$ axis, xy-plane, and xz-plane (shown in Figure 7 (a-d)). The current fluctuations are because of the alterations of bases' geometry and orientations, and lateral translation. The figure displays the current for nitrogen passivated nanopore at $300 \mathrm{~K}$. Equation (3) is used to calculate the current by integrating the transmission spectra. Figure 11 displays the current ranges because of each base rotation at $2.8 \mathrm{~V}$ bias within the $\mathrm{N}$-pore sensor. The figure shows unique current signature for the different DNA bases positioned within the N-pore. It shows the intervals that have to be set as the current variations limits. The work shows that each DNA base has a unique signature Moreover, the figure shows that pyrimidine bases have lower current than purine bases because of the physical and chemical structures of the DNA bases which makes it possible to identify the two different categories of DNA nucleobases at a fixed bias voltage. The reason for the higher current of purine bases compared with pyrimidine is that purine consists of two hydrogen-carbon rings and four nitrogen atoms, while pyrimidines consist of one hydrogen-carbon ring and two nitrogen atoms. Graphene nanopore edge functionalization modifies the sensor behavior. Our work agrees with $[50,51]$ where the nitrogen passivation of the nanopore and nanogap improves the sensitivity when compared to hydrogen passivation. Moreover, the lowest to highest order of the DNA bases transmission and current is the same due to both types of passivation, but the transmission and current are higher with $\mathrm{N}$-pore $[50,51]$. Also, it was noticed that the tunneling pore or gap is bridged in a better way with purine bases resulting in a higher current for purine bases in comparison to pyrimidine bases [51]. The overall transmittance is highly increased in nitrogen termination. In particular, the electrical current is larger and easier to detect due to $\mathrm{N}$-functionalization. The sensor with N-pore has stronger interaction and better coupling between the DNA base and the sensor and this fact is because of the hybridization between the DNA bases and the states from the edge leading to higher charge transfer.

In experiment, the DNA strand will be pulled through the nanopore within the graphene nanoribbon while a voltage will be applied between the left and right electrodes. Each of the different DNA bases is expected to modify the current in a unique way which makes it possible to differentiate the DNA bases. The designed sensor should be able to detect the sequence, but this means that the speed of DNA passing through the pore should be controlled precisely.

Theoretical work proofed that N-terminated pores enhance the single biomolecule detection and makes the sensor highly sensitive [43-45]. Most of the theoretical work conducted with nanoribbons was generated with simple model systems where the effect of solvent and ions are not considered [30, 50, 52]. The generated transverse current for each DNA base is expected to be higher than the effect of the solvent $[11,50$, 52].

The variation in current for the DNA bases is more pronounced when the pore is terminated by nitrogen. The nitrogen passivation results in higher current readings and higher variations in the current between the different DNA bases, as shown in Figure 8, which enhances the sensor sensitivity [30]. Such current is expected to be higher than the resulting electronic noise caused by DNA fluctuations during translocation [30]. Moreover, the large operating current may remove the necessity for slowing down the DNA translocation since the speed of measuring the current is expected to be high enough to avoid Brownian fluctuations from blurring the signal. 

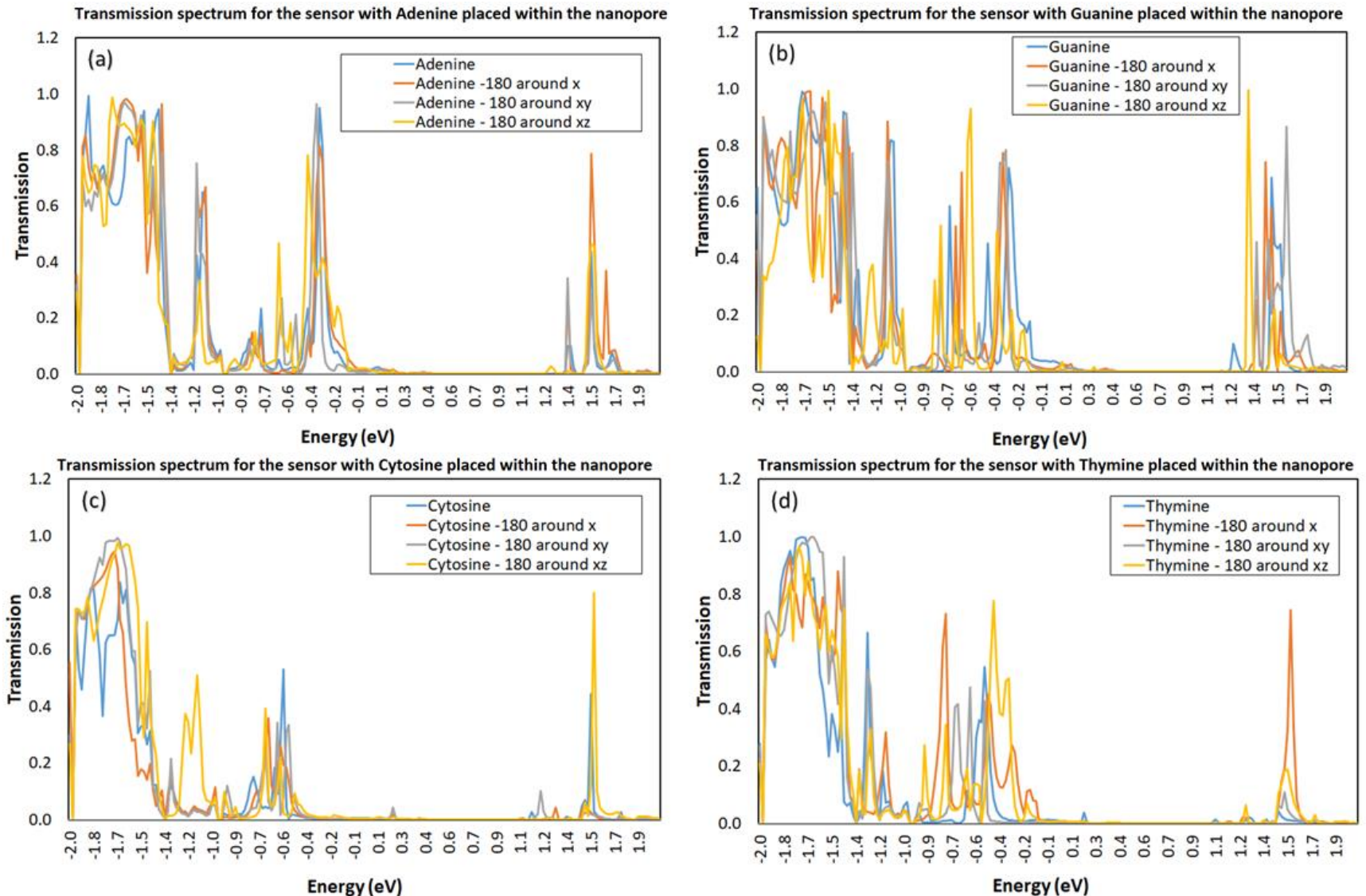

Figure 5. The transmission spectra for the different types of DNA nucleobases at a bias voltage of $2.8 \mathrm{~V}$. The different colors represent the different orientations for each DNA base.

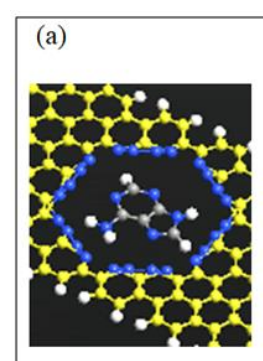

Adenine (b)

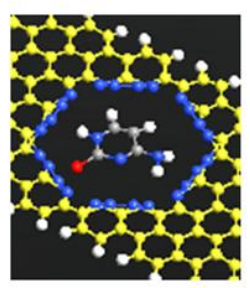

Cytosine (c)

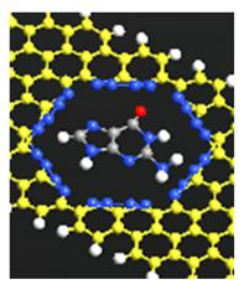

Guanine (d)

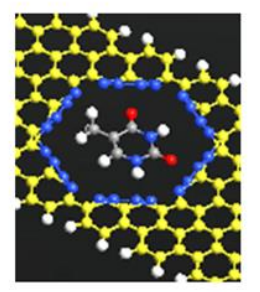

Thymine

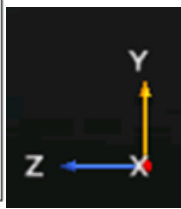

Figure 6. The structure of the nanopores with different types of DNA bases (A, G, C, and T) at $0^{\circ}$ orientation.

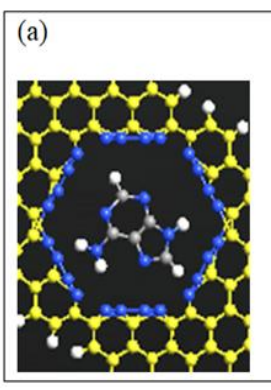

(b)

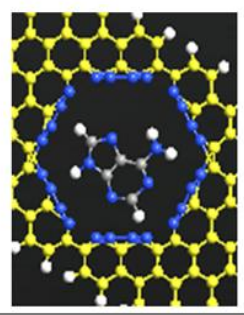

(c)

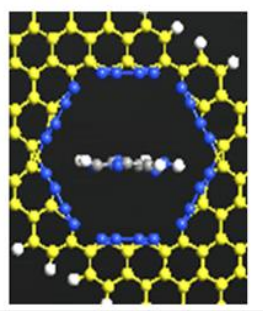

(d)

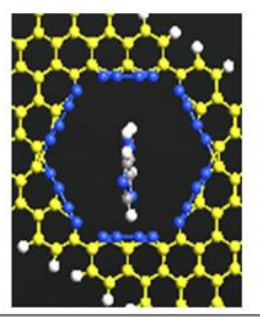

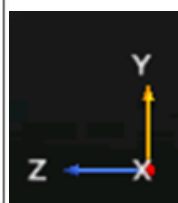

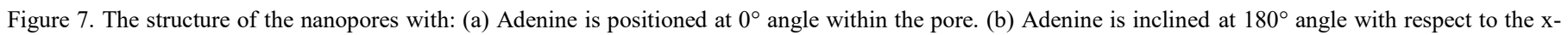
axis. (c) Adenine is inclined at $180^{\circ}$ angle with respect to the xy-plane. (d) Adenine is inclined at $180^{\circ}$ angle with respect to the xz-plane. 


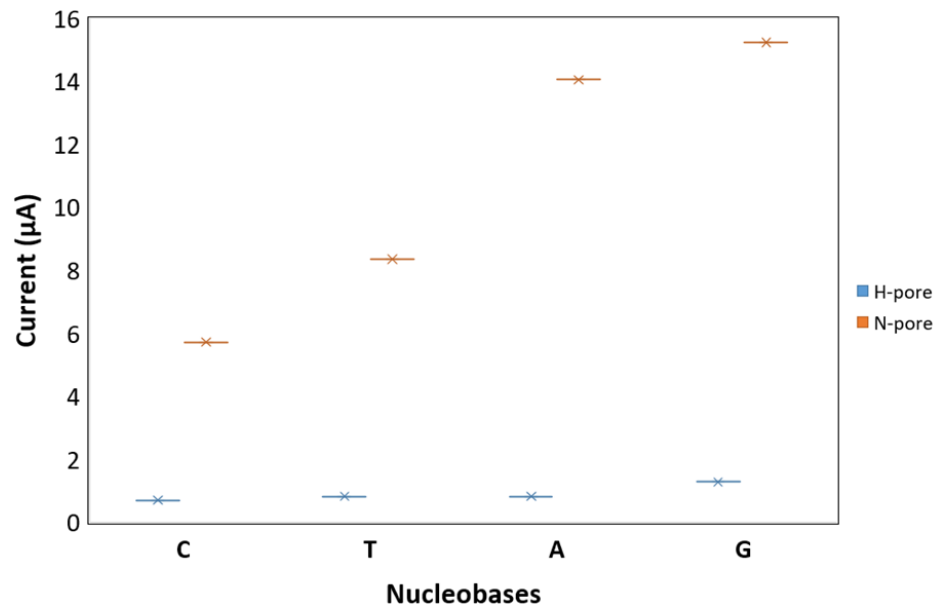

Figure 8. Current of the z-shaped device when each of the DNA nucleobases is placed into the middle of the N-pore or H-pore with $\sim 1.01 \mathrm{~nm}$ diameter.

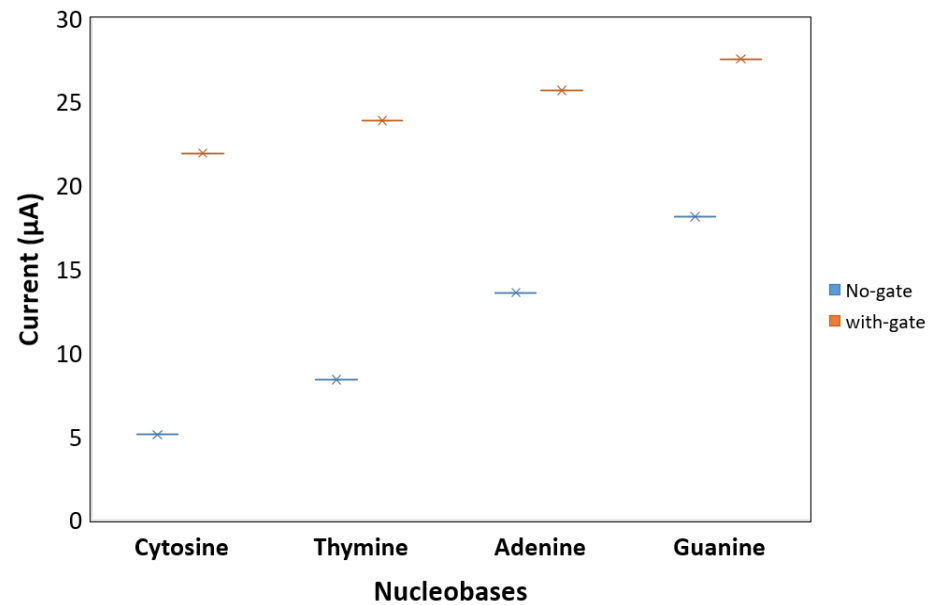

Figure 9. Current of the z-shaped device with and without gate voltage when each of the DNA nucleobases is positioned into the middle of the N-pore with $\sim 1.01 \mathrm{~nm}$ diameter.

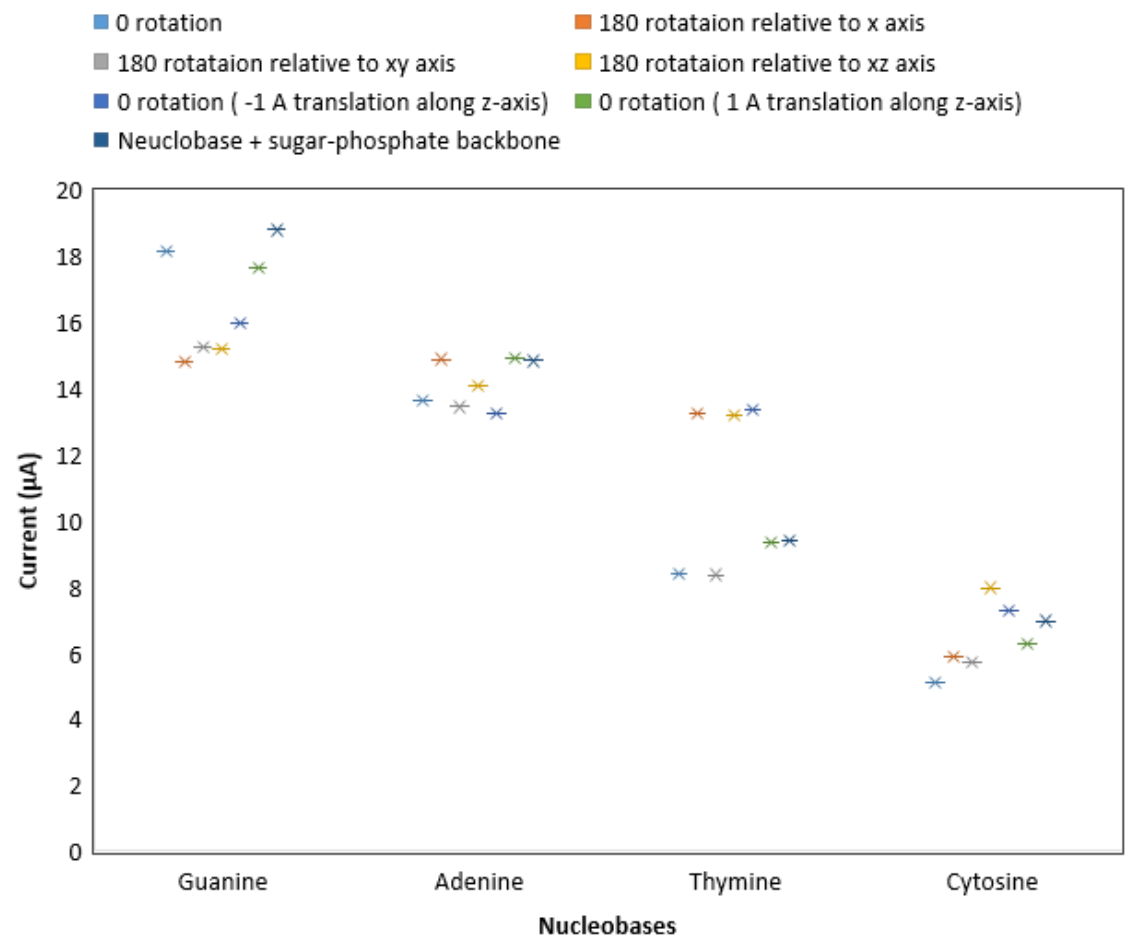

Figure 10. Current difference due to nucleobase various orientations, lateral translation, and addition of DNA backbone in the z-shaped sensor nanopore. 


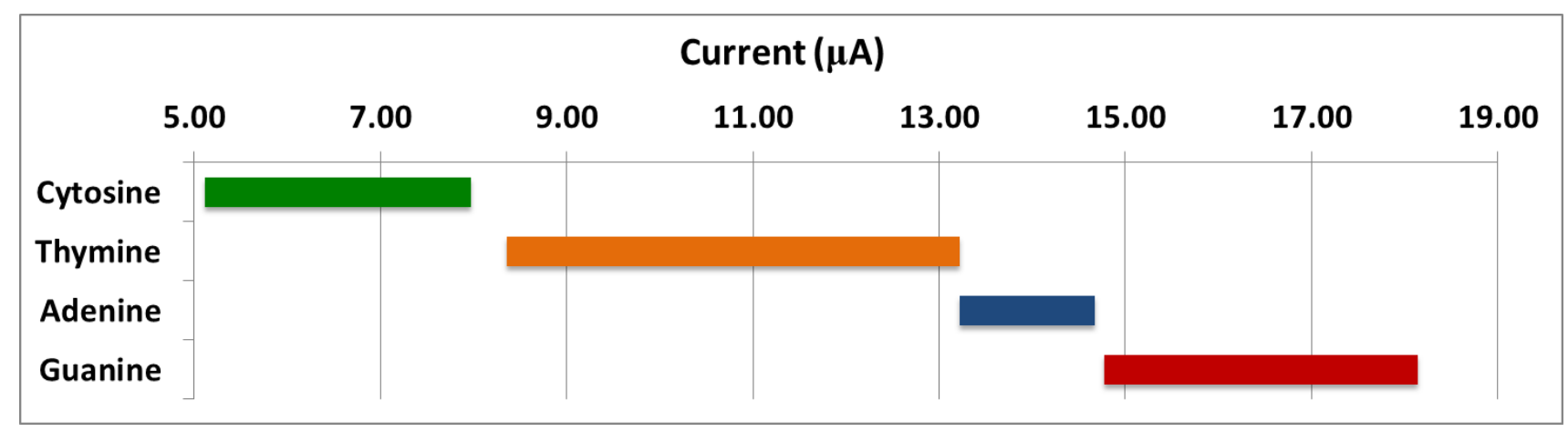

Figure 11. Current ranges resulting from Neuclobases' orientations, translations, and addition sugar-phosphate backbone at $2.8 \mathrm{~V}$ bias in the N-pore.

Intrinsic stepwise translocation for single stranded DNA (ssDNA) through a graphene pore can be used to improve the signal readings to identify the DNA nucleotides [53-56]. The stepwise translocation can be accomplished by stretching the ssDNA mechanically while passing through the graphene pore $[53,54]$. Qiu et al. found that ssDNA stepwise motion can be accomplished and helps to accurately identify the DNA nucleotides' passing through graphene pore. A harmonic spring was moved at a fixed velocity while the other end was attached to all phosphorus atoms which helps in preventing the tension between neighboring nucleotides [53]. The force applied to the spring varies due to the adhesive interaction among the DNA bases and graphene membrane. Moreover, mechanical stretching of DNA can prevent backward movement of DNA.

In our study, graphene was considered as a rigid structure and DNA passed through the pore with various orientations and lateral translation. This can be achieved in experiment by applying a force to pull DNA backbone to overcome hydrophobic interactions between graphene and DNA nucleotides. The mechanical stepwise technique is promising and helps to enhance the signal, slow down the translocation, reduce noise, and stabilize the DNA bases [53].

\section{Noise factors}

Various factors may cause electronic noise that affects the possibility of differentiating the DNA bases. These factors are anticipated to result in small noise on top of the large current of the z-shaped sensor. This is confirmed by studying some of these factors effect on transmission spectrum and current:

\section{1) DNA backbone of sugar and phosphate group}

The DNA backbone consisting of sugar and phosphate group is attached to the DNA nucleobases which impacts the current modulation. However, this effect is expected to be small as shown in Figure 12 and enclosed by the intervals displayed in Figure 11.

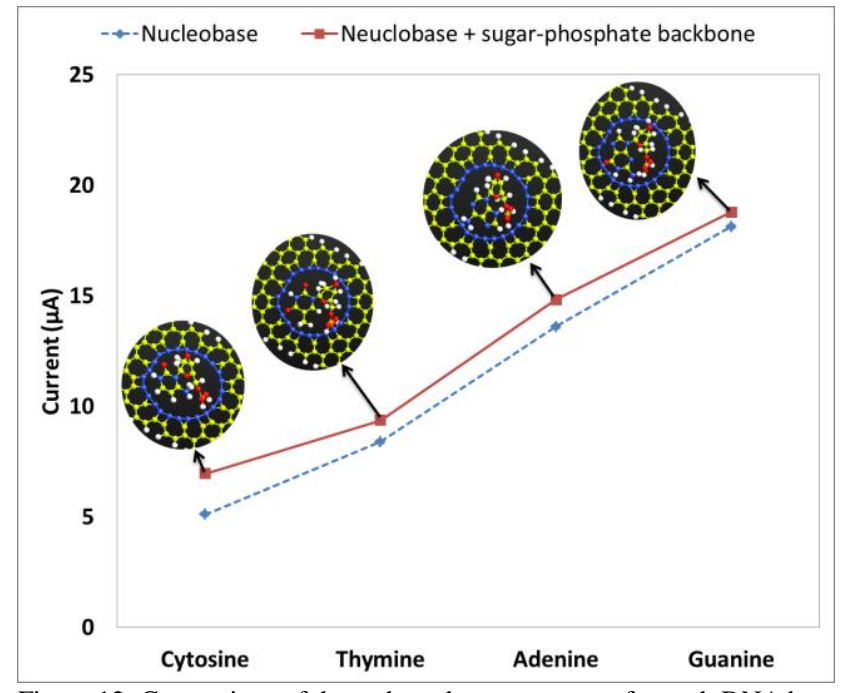

Figure 12: Comparison of the $z$-shaped sensor current for each DNA base positioned in the middle of the N-pore and for the DNA bases after they are attached to sugar-phosphate backbone.

The sugar-phosphate backbone can be processed as systematic noise that can be identified and removed as confirmed by previous studies $[57,58]$. This simplification is due to the possibility of separating the DNA bases signal from noise by deducting the noise generated from the DNA backbone.

\section{2) DNA Nucleobases' translation}

It is important to estimate the cases where the positions of the DNA bases are changed in the radial direction of the pore, which usually happens in real experiments. The transmission spectrum due to the DNA bases translation effect is displayed in Figure 14 where each base is translated $1 \AA$ to the right direction and $1 \AA$ to the left direction as displayed in Figure 13. (a)

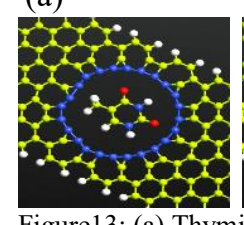

(b)

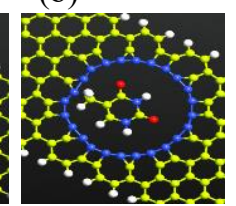

(c)

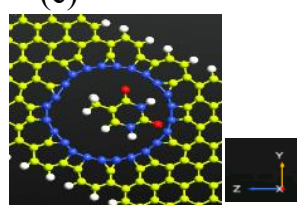

Figure13: (a) Thymine within the pore at $0^{\circ}$ angle. (b) Thymine due to -1 $\AA$ translation along the z-axis. (c) Thymine due to $1 \AA$ translation along the z-axis. 

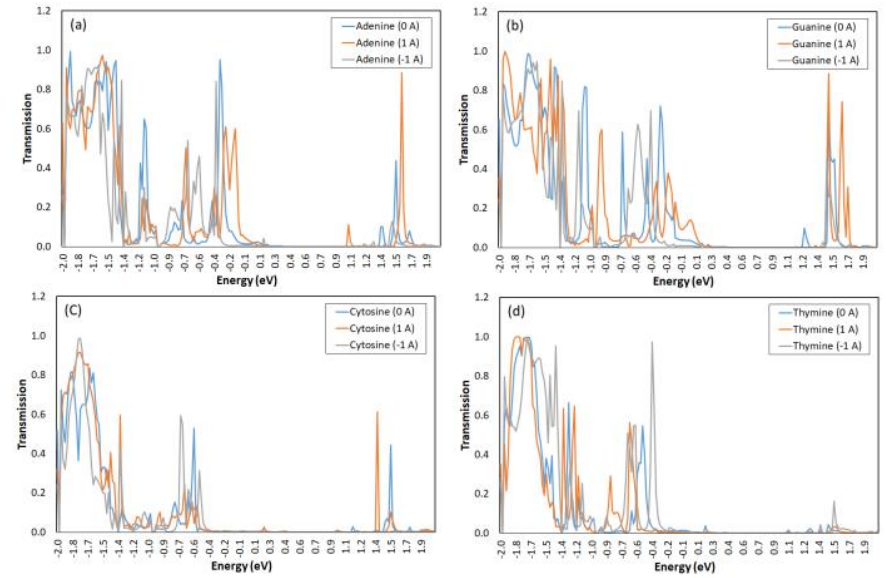

Figure 14: The transmission spectra change due to $\pm 1.0 \AA$ translation along the z-axis for: (a) Adenine, (b) Guanine, (c) Cytosine, and (d) Thymine. The transmission spectra colors refer to the base orientation within the sensor nanopore

The effect of lateral translation of each nucleobase is displayed in Figure 10. The transmission spectrum is slightly affected by the translation which resulted in slight changes in the current within the current intervals displayed in Figure 11.

\section{3) Nearest Neighbor}

Figure 15 shows the nearest neighbor effect on the current of deoxycytidine monophosphate (dCMP) where a second nucleotide such as deoxycytidine monophosphate (dCMP) or deoxythymidine monophosphate (dTMP) was placed on top of the first one. The current is generated when dCMP has another dCMP on top of it and when dCMP has dTMP on top of it. The distance between each two nucleotides is $3 \AA$. The variations in current are small as shown in Figure 15 and within the intervals of Figure 11. A spatial separation of more than $2 \AA$ between the nanopore and the second nucleotide is enough to remove its direct contributions from the nucleotide molecular orbitals to the sensor current as confirmed by previous studies [59].

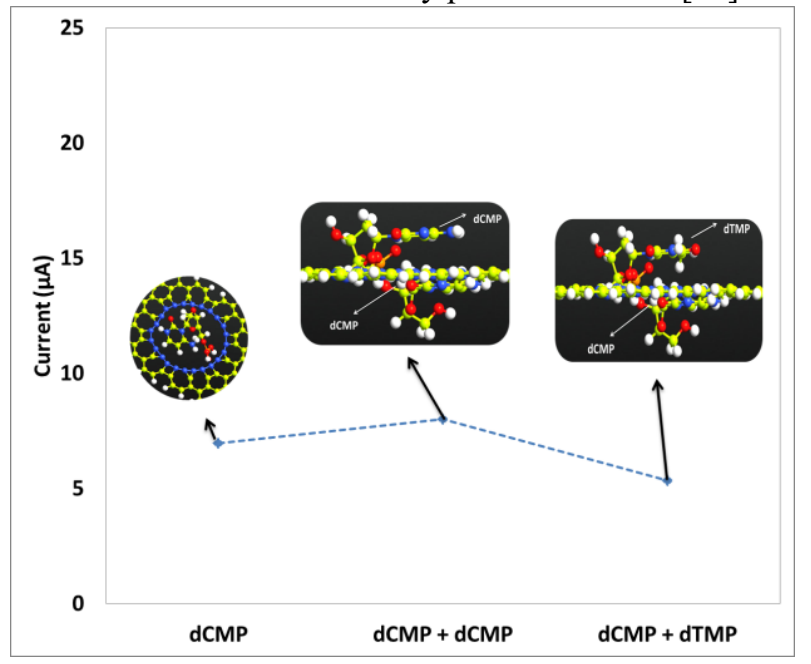

Figure 15: Comparison of the z-shaped sensor current for (dCMP) in the middle of the N-pore and when dCMP has another dCMP on top of it or dCMP has dTMP on top of it.

\section{4) Solution}

Figure 16 illustrates how the presence of a water molecule affects the sensor current. The change in current is slight approximately $0.3 \mu \mathrm{A}$. The transverse current is expected to be much larger than the dynamical environment effect on the neuclotides electronic structure in solution $[30,52,60]$.

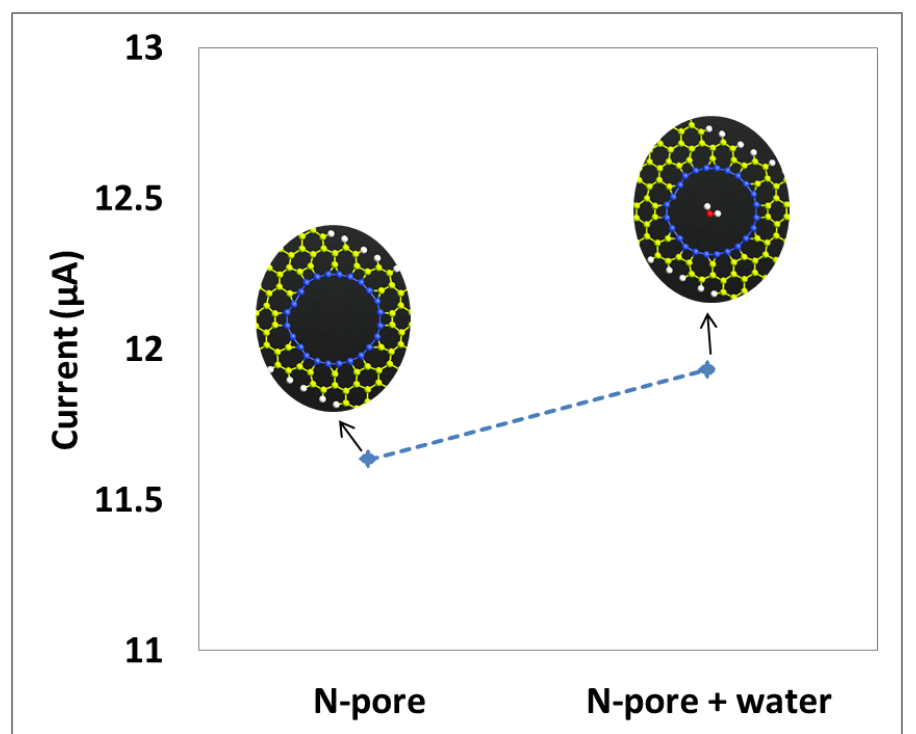

Figure 16: The z-shaped sensor current when the N-pore is empty and when a water molecule is placed within the pore.

Thus, all the previously mentioned noise factors are encompassed by the intervals' boundaries in Figure 11. Moreover, it is worth noting that QuantumATK doesn't supply computational details for DNA translocation within pores [30, 52, 59]. Di Ventra et al. [61] found that the electronic noise such as DNA motion and fluctuations while measuring the transverse tunneling current would likely result in small noise. This noise will not affect the current distributions of the different DNA nucleotides and the ability to identify each base.

\section{DISCUSSION}

Intensive research work was conducted using transverse current DNA sensors with a nanopore and nanogap where nucleobases (or nucleotides) were placed in a pore or gap and rotated by up to 180 degrees to study their range of current response $[30,52]$. These researches resulted in unique current for the different DNA bases. However, these studies resulted in small current and conductance. Moderate improvements were offered by various theoretical studies to enhance the electrical current of DNA in nanogaps $[24,62,63]$ and nanopores $[19,39,59]$. The designed sensor is enhanced by using nitrogen instead of hydrogen to passivate edge carbon atoms. The edge termination of graphene nanoribbons by nitrogen is noticed to be rich of electrons leading to n-type transistor behavior [43]. The edge structure and chemical termination is critical to get the desired device characteristics. It is noticed that nitrogen passivated graphene pore sensors are very sensitive to intramolecular electrostatics [43]. Previous work [45] confirms the improved sensitivity of nitrogen 
passivation in comparison to hydrogen passivation. The transverse current magnitude of the sensor is enhanced since the nitrogen bonds enhance the coupling between the sensor and the DNA bases. De Souza et al. designed a novel sensor using a hybrid sheet of graphene and hexagonal boron nitride with a nanopore to distinguish among the different DNA bases where each DNA base with distinct dipole modifies that charge uniquely and results in a unique current [64]. His work showed significant mechanism to sense DNA that relies on shifting the chemical potential of the sensor by applying a specific gate potential [64]. The sensor sensitivity is associated with the gate potential value and the applied bias voltage [40, $50,64]$. Gate potential controls the local current path within the sensor [50]. Our current work indicates that the designed sensor is required to be set to specific gate potential and bias voltage to get unique signature for the four DNA bases with high sensitivity. In the designed z-shaped sensor, the optimal results were generated when the dual gate was biased at $1 \mathrm{~V}$ each side and the bias potential was fixed among the two electrodes $(+1.4$ and $-1.4 \mathrm{eV})$.

Moreover, adding a gate and applying a gate voltage to the Z-shaped nanoribbon improves the sensor sensitivity and gives higher transverse current by shifting the Fermi energy towards the conduction band from the charge neutrality point. The gate electrode is placed very close to the semiconducting channel so that the applied electric charge affects the channel. The gate can control the carriers flow (holes or electrons) passing between source and drain. Applying a gate voltage increases the number of carriers within the semiconducting channel, which leads to higher current between source and drain. The utilization of $\mathrm{N}$-pore and adding a gate terminal makes it feasible to measure, detect, and read the electrical properties of DNA bases. Each DNA base affects the charge density in a unique way resulting in a significant electronic signature.

\section{CONCLUSION}

Sensors based on nanopore are promising in the identification of the DNA sequence inexpensively and rapidly without the necessity for amplification or labeling. Electronic transport simulations are generated using SC-EH+NEGF formalism to study the bases' signal within a pore hosted by the developed $\mathrm{z}$-shaped graphene nanoribbon device.

This study shows a theoretical analysis of electronic transport in the developed transistor with a pore based on the various DNA nucleobases. Each DNA base generates a specific signature because of its unique electronic and chemical structure. Pyrimidine nucleobases result in lower current than purine nucleobases. The current modifications due to the bases' orientation are studied at fixed bias voltage. Despite the current changes because of the base orientations, a unique signal for each DNA base is possible. These signals or signatures change slightly because of the bases' orientations within a pore.

The sensor sensitivity is enhanced by using nitrogen instead of hydrogen to passivate the pore edge carbon atoms. Passivation can be accomplished by nitrogen or hydrogen, where the nitrogen passivation results in a sensor with excess free electrons working as an n-type device. Moreover, adding a dual gate terminal to $\mathrm{z}$-shaped nanoribbon and applying a gate potential improved the sensor reading and signal.

\section{REFERENCES}

[1] H. Lehrach, "DNA sequencing methods in human genetics and disease research," F1000Prime Rep, vol. 5, pp. 34, Sep 2, 2013.

[2] Z. Mingjun, C. L. Sabharwal, T. Weimin, T. Tzyh-Jong, X. Ning, and L. Guangyong, "Interactive DNA sequence and structure design for DNA nanoapplications," IEEE Transactions on NanoBioscience, vol. 3, no. 4, pp. 286-292, 2004.

[3] A. Wasfi, F. Awwad, and A. I. Ayesh, "Graphene-based nanopore approaches for DNA sequencing: A literature review," Biosensors and Bioelectronics, vol. 119, pp. 191-203, 2018/11/15/, 2018.

[4] G. Church, and W. Deamer David, Characterization of individual polymer molecules based on monomer-interface interactions, to PRESIDENT AND FELLOWS OF HARVARD COLLEGE, 1996.

[5] B. M. Venkatesan, and R. Bashir, "Nanopore sensors for nucleic acid analysis," Nature Nanotechnology, vol. 6, pp. 615, 09/18/online, 2011.

[6] M. Zwolak, and M. Di Ventra, "Electronic Signature of DNA Nucleotides via Transverse Transport," Nano Letters, vol. 5, no. 3, pp. 421-424, 2005/03/01, 2005.

[7] J. Lagerqvist, M. Zwolak, and M. Di Ventra, "Fast DNA Sequencing via Transverse Electronic Transport," Nano Letters, vol. 6, no. 4, pp. 779-782, 2006/04/01, 2006.

[8] M. Di Ventra, "Fast DNA sequencing by electrical means inches closer," Nanotechnology, vol. 24, no. 34, pp. 342501, 2013/07/30, 2013.

[9] M. Di Ventra, M. Krems, J. Wilson, and Y. Pershin, "DNA Characterization by Transverse Electrical Current in a Nanochannel," Methods in molecular biology (Clifton, N.J.), vol. 870, pp. 149-63, 02/29, 2012.

[10] P. Krstić, B. Ashcroft, and S. Lindsay, "Physical model for recognition tunneling," Nanotechnology, vol. 26, no. 8, pp. 084001, 2015/02/03, 2015.

[11] D. Branton, D. W. Deamer, A. Marziali, H. Bayley, S. A. Benner, T. Butler, M. Di Ventra, S. Garaj, A. Hibbs, X. Huang, S. B. Jovanovich, P. S. Krstic, S. Lindsay, X. S. Ling, C. H. Mastrangelo, A. Meller, J. S. Oliver, Y. V. Pershin, J. M. Ramsey, R. Riehn, G. V. Soni, V. Tabard-Cossa, M. Wanunu, M. Wiggin, and J. A. Schloss, "The potential and challenges of nanopore sequencing," Nature Biotechnology, vol. 26, pp. 1146, 10/09/online, 2008.

[12] S. Lindsay, J. He, O. Sankey, P. Hapala, P. Jelinek, P. Zhang, S. Chang, and S. Huang, "Recognition tunneling," Nanotechnology, vol. 21, no. 26, pp. 262001-262001, 2010.

[13] E. Paulechka, T. A. Wassenaar, K. Kroenlein, A. Kazakov, and A. Smolyanitsky, "Nucleobase-functionalized graphene nanoribbons for accurate high-speed DNA sequencing," Nanoscale, vol. 8, no. 4, pp. 1861-7, Jan 28, 2016.

[14] E. Sheka, "The Uniqueness of Physical and Chemical Natures of Graphene: Their Coherence and Conflicts," International Journal of Quantum Chemistry, vol. 114, 08/15, 2014.

[15] S. J. Heerema, and C. Dekker, "Graphene nanodevices for DNA sequencing," Nat Nanotechnol, vol. 11, no. 2, pp. 127-36, Feb, 2016.

[16] C. Fermanian Kammerer, and F. Méhats, "A kinetic model for the transport of electrons in a graphene layer," Journal of Computational Physics, vol. 327, pp. 450-483, 2016.

[17] X. Lu, J. Yvonnet, F. Detrez, and J. Bai, "Multiscale modeling of nonlinear electric conductivity in graphene-reinforced nanocomposites taking into account tunnelling effect," Journal of Computational Physics, vol. 337, pp. 116-131, 2017.

[18] K. Wakabayashi, Y. Takane, M. Yamamoto, and M. Sigrist, "New Journal of Physics Electronic transport properties of graphene nanoribbons," New Journal of Physics, vol. 11, pp. 95016-21, 09/01, 2009

[19] W. Qiu, P. Nguyen, and E. Skafidas, "Graphene nanopores: electronic transport properties and design methodology," Phys Chem Chem Phys, vol. 16, no. 4, pp. 1451-9, Jan 28, 2014.

[20] J. Prasongkit, G. T. Feliciano, A. R. Rocha, Y. He, T. Osotchan, R. Ahuja, and R. H. Scheicher, "Theoretical assessment of feasibility to sequence DNA through interlayer electronic tunneling transport 
at aligned nanopores in bilayer graphene," Sci Rep, vol. 5, pp. 17560, Dec 4, 2015.

[21] S. Garaj, W. Hubbard, A. Reina, J. Kong, D. Branton, and J. A. Golovchenko, "Graphene as a subnanometre trans-electrode membrane," Nature, vol. 467, pp. 190, 08/18/online, 2010.

[22] C. A. Merchant, K. Healy, M. Wanunu, V. Ray, N. Peterman, J. Bartel, M. D. Fischbein, K. Venta, Z. Luo, A. T. Johnson, and M. Drndic, "DNA translocation through graphene nanopores," Nano Lett, vol. 10, no. 8, pp. 2915-21, Aug 11, 2010.

[23] G. F. Schneider, S. W. Kowalczyk, V. E. Calado, G. Pandraud, H. W. Zandbergen, L. M. K. Vandersypen, and C. Dekker, "DNA Translocation through Graphene Nanopores," Nano Letters, vol. 10, no. 8, pp. 3163-3167, 2010/08/11, 2010.

[24] H. W. Postma, "Rapid sequencing of individual DNA molecules in graphene nanogaps," Nano Lett, vol. 10, no. 2, pp. 420-5, Feb 10, 2010

[25] R. Emadi, R. Safian, A. Z. Nezhad, and R. Emadi, "Analysis and Design of Graphene-Based Surface Plasmon Waveguide Switch at Long-Wavelength Infrared Frequencies," IEEE Journal of Selected Topics in Quantum Electronics, vol. 23, no. 5, pp. 1-9, 2017.

[26] Y. Wang, and D. R. Andersen, "Quantum Size Effects in the Terahertz Nonlinear Response of Metallic Armchair Graphene Nanoribbons," IEEE Journal of Selected Topics in Quantum Electronics, vol. 23, no. 1, pp. 148-155, 2017.

[27] F. Yan, M. Zhang, and J. Li, "Solution-Gated Graphene Transistors for Chemical and Biological Sensors," Advanced Healthcare Materials, vol. 3, no. 3, pp. 313-331, 2014.

[28] M. Puster, J. A. Rodríguez-Manzo, A. Balan, and M. Drndić "Toward Sensitive Graphene Nanoribbon-Nanopore Devices by Preventing Electron Beam-Induced Damage," ACS Nano, vol. 7, no. 12, pp. 11283-11289, 2013/12/23, 2013.

[29] S. K. Min, W. Y. Kim, Y. Cho, and K. S. Kim, "Fast DNA sequencing with a graphene-based nanochannel device," Nature Nanotechnology, vol. 6, pp. 162, 02/06/online, 2011.

[30] K. K. Saha, M. Drndić, and B. K. Nikolić, "DNA Base-Specific Modulation of Microampere Transverse Edge Currents through a Metallic Graphene Nanoribbon with a Nanopore," Nano Letters, vol. 12, no. 1, pp. 50-55, 2012/01/11, 2012.

[31] F. Traversi, C. Raillon, S. M. Benameur, K. Liu, S. Khlybov, M. Tosun, D. Krasnozhon, A. Kis, and A. Radenovic, "Detecting the translocation of DNA through a nanopore using graphene nanoribbons," Nature Nanotechnology, vol. 8, pp. 939, 11/17/online, 2013.

[32] A. Wasfi, F. Awwad, and A. I. Ayesh, "DNA sequencing via Zshaped graphene nano ribbon field effect transistor decorated with nanoparticles using first-principle transport simulations," New Journal of Physics, vol. 22, no. 6, pp. 063004, 2020/06/15, 2020.

[33] J. Taylor, H. Guo, and J. Wang, "Ab initio modeling of quantum transport properties of molecular electronic devices," Phys. Rev. B, vol. 63, 06/01, 2001.

[34] M. Brandbyge, J.-L. Mozos, P. Ordejón, J. Taylor, and K. Stokbro, "Density-functional method for nonequilibrium electron transport," Physical Review B, vol. 65, no. 16, pp. 165401, 03/22/, 2002.

[35] J. Cerdá, and F. Soria, "Accurate and transferable extended Hl"uckel-type tight-binding parameters," Physical Review B, vol. 61, no. 12, pp. 7965-7971, 03/15/, 2000.

[36] D. A. Papaconstantopoulos, and M. J. Mehl, "The Slater Koster tight-binding method: a computationally efficient and accurate approach," Journal of Physics: Condensed Matter, vol. 15, no. 10, pp. R413-R440, 2003/03/03, 2003.

[37] K. Stokbro, D. Petersen, S. Smidstrup, A. Blom, M. Ipsen, and K. Kaasbjerg, "Semi-Empirical Model for Nano-Scale Device Simulations," Physical Review B, vol. 82, 04/16, 2010.

[38] A. Wasfi, and F. Awwad, "Semi-empirical Modeling for DNA Bases via Z-shaped Graphene Nanoribbon with a Nanopore." pp. 16-21.

[39] A. Wasfi, F. Awwad, and A. I. Ayesh, "Electronic signature of DNA bases via Z-shaped graphene nanoribbon with a nanopore," Biosensors and Bioelectronics: X, pp. 100011, 2019.

[40] B. P. Pandey, "Transmission spectrum and IV characteristics of dual-gate Z-shaped graphene nanoribbon FET," Nanomaterials and Energy, vol. 7, no. 2, pp. 32-36, 2018.

[41] F. Zahid, M. Paulsson, E. Polizzi, A. Ghosh, L. Siddiqui, and S. Datta, "A self-consistent transport model for molecular conduction based on extended Hückel theory with full three-dimensional electrostatics," The Journal of chemical physics, vol. 123, pp. 64707, 09/01, 2005.

[42] D. Kienle, J. I. Cerda, and A. W. Ghosh, "Extended Hückel theory for band structure, chemistry, and transport. I. Carbon nanotubes," Journal of Applied Physics, vol. 100, no. 4, pp. 043714, 2006.

[43] F. Al-Dirini, M. A. Mohammed, M. S. Hossain, F. M. Hossain, A. Nirmalathas, and E. Skafidas, "Tuneable graphene nanopores for single biomolecule detection," Nanoscale, vol. 8, no. 19, pp. 10066-77, May 21, 2016

[44] X. Wang, X. Li, L. Zhang, Y. Yoon, P. K. Weber, H. Wang, J. Guo, and H. Dai, "N-doping of graphene through electrothermal reactions with ammonia," Science, vol. 324, no. 5928, pp. 768-71, May 8, 2009.

[45] F. Al-Dirini, F. M. Hossain, A. Nirmalathas, and E. Skafidas, "AllGraphene Planar Self-Switching MISFEDs, Metal-InsulatorSemiconductor Field-Effect Diodes," Scientific Reports, vol. 4, no. 1, pp. 3983, 2014/02/05, 2014.

[46] J. P. Perdew, K. Burke, and M. Ernzerhof, "Generalized Gradient Approximation Made Simple," Physical Review Letters, vol. 77, no. 18 , pp. 3865-3868, 10/28/, 1996.

[47] V. Narendar, S. K. Gupta, and S. Saxena, "First Principle Study of Doped Graphene for FET Applications," Silicon, vol. 11, no. 1, pp. 277-286, 2018.

[48] M. Di Ventra, Electrical Transport in Nanoscale Systems, Cambridge: Cambridge University Press, 2008.

[49] P.-H. Chang, H. Liu, and B. Nikolic, "First-principles vs. semiempirical modeling of global and local electronic transport properties of graphene nanopore-based sensors for DNA sequencing," Journal of Computational Electronics, vol. 13, 08/19, 2014.

[50] F. Leão de Souza, R. Amorim, W. Scopel, and R. Scheicher, "Controlled current confinement in interfaced 2D nanosensor for electrical identification of DNA," Physical Chemistry Chemical Physics, vol. 21, 09/23, 2019.

[51] R. G. Amorim, A. R. Rocha, and R. H. Scheicher, "Boosting DNA Recognition Sensitivity of Graphene Nanogaps through Nitrogen Edge Functionalization," The Journal of Physical Chemistry C, vol. 120, no. 34, pp. 19384-19388, 2016.

[52] J. Prasongkit, A. Grigoriev, B. Pathak, R. Ahuja, and R. H. Scheicher, "Transverse Conductance of DNA Nucleotides in a Graphene Nanogap from First Principles," Nano Letters, vol. 11, no. 5, pp. 1941-1945, 2011/05/11, 2011.

[53] H. Qiu, A. Sarathy, J. P. Leburton, and K. Schulten, "Intrinsic Stepwise Translocation of Stretched ssDNA in Graphene Nanopores," Nano Lett, vol. 15, no. 12, pp. 8322-30, Dec 9, 2015.

[54] H. Qiu, A. Sarathy, J.-P. Leburton, and K. Schulten, "Stepwise Transport of Stretched ssDNA Through Graphene Nanopores," Biophysical Journal, vol. 110, no. 3, pp. 508a, 2016.

[55] B. Luan, D. Wang, R. Zhou, S. Harrer, H. Peng, and G. Stolovitzky, "Dynamics of DNA translocation in a solid-state nanopore immersed in aqueous glycerol," Nanotechnology, vol. 23, no. 45, pp. 455102, 2012/10/12, 2012.

[56] B. Luan, H. Peng, S. Polonsky, S. Rossnagel, G. Stolovitzky, and G. Martyna, "Base-by-base ratcheting of single stranded DNA through a solid-state nanopore," Physical review letters, vol. 104, no. 23, pp. 238103, 2010/06//, 2010.

[57] H. L. McFarland, T. Ahmed, J. X. Zhu, A. V. Balatsky, and J. T. Haraldsen, "First-Principles Investigation of Nanopore Sequencing Using Variable Voltage Bias on Graphene-Based Nanoribbons," $J$ Phys Chem Lett, vol. 6, no. 13, pp. 2616-21, Jul 2, 2015.

[58] T. Ahmed, J. T. Haraldsen, J. J. Rehr, M. Di Ventra, I. Schuller, and A. V. Balatsky, "Correlation dynamics and enhanced signals for the identification of serial biomolecules and DNA bases," Nanotechnology, vol. 25, no. 12, pp. 125705, Mar 28, 2014.

[59] T. Nelson, B. Zhang, and O. Prezhdo, "Detection of Nucleic Acids with Graphene Nanopores: Ab Initio Characterization of a Novel Sequencing Device," Nano letters, vol. 10, pp. 3237-42, 09/08, 2010.

[60] H. Postma, "Rapid Sequencing of Individual DNA Molecules in Graphene Nanogaps," Nano letters, vol. 10, pp. 420-5, 02/10, 2010.

[61] M. Krems, M. Zwolak, Y. Pershin, and M. Di Ventra, Effect of Noise on DNA Sequencing via Transverse Electronic Transport, 2009. 
[62] Y. He, R. Scheicher, A. Grigoriev, R. Ahuja, S. Long, Z. Huo, and M. Liu, "Enhanced DNA Sequencing Performance Through Edge-Hydrogenation of Graphene Electrodes," Advanced Functional Materials, vol. 21, pp. 2674-2679, 07/22, 2011.

[63] M. Tsutsui, M. Taniguchi, K. Yokota, and T. Kawai, "Identifying single nucleotides by tunnelling current," Nature Nanotechnology, vol. 5, pp. 286, 03/21/online, 2010.

[64] F. A. de Souza, R. G. Amorim, W. L. Scopel, and R. H. Scheicher, "Electrical detection of nucleotides via nanopores in a hybrid graphene/h-BN sheet," Nanoscale, vol. 9, no. 6, pp. 2207-2212, Feb 9, 2017.

Asma Wasfi: received her Master degree in Electrical Engineering from United Arab Emirates Univresity. She is currently continuing her Ph.D. in the Department of Electrical Engineering, United Arab Emirates University.

Her research interests include graphene nanoribbon, DNA detection, and sensor fabrication.

Prof. Falah Awwad: Professor, Department of Electrical Engineering, United Arab Emirates University, Al Ain, United Arab Emirates

Prof. Falah Awwad received the M.A.Sc. and Ph.D. degrees in Electrical and Computer Engineering from Concordia University (Montreal, QC, Canada) in 2002 and 2006, respectively. He was a Post-Doctoral Fellow at Ecole Polytechnique de Montréal and Concordia University, Montreal, QC, Canada. Between August 2007 - Feb. 2013, he was an Assistant Professor with the College of Information Technology (CIT) at United Arab Emirates University. Currently, he is a Professor with the Department of Electrical EngineeringCollege of Engineering (UAE University). He published over 80 research articles in peer-reviewed journals and international conferences. He is a member of the editorial boards of several Journals. He is a regular reviewer of many journals including IEEE TVLSI, TCAS-I, etc. He is the PI of 20 projects and supervised several postgraduate students. His scientific research interests include primarily sensors, circuits, and devices, in addition to VLSI, hardware security, and biomedical applications.

Prof. Ahmad I. Ayesh: Director of Center for Sustainable Development and Professor of Physics at the Math, Stat. and Physics Department, Qatar University, Doha, Qatar.

Prof. Ayesh is the leader of the Nanomaterial Synthesis and their Devices Applications group at Qatar University. He is expert in the nano- and microdevice fabrication using both the top-down and bottom-up approaches as well as the self-assembly of the nanostructure within the device. Furthermore, he is expert in nanomaterial fabrication and characterization. Prof. Ayesh has 101 publications in refereed international journals and conferences. 